\title{
FAMILIA E INVESTIGACIÓN: ESTRATEGIAS FAMILIARES Y DE GÉNERO DE LAS INVESTIGADORAS ESPAÑOLAS
}

\author{
ANA GONZÁlEZ RAMOS \\ Internet Interdisciplinary Institute (IN3), Universitat Oberta de Catalunya \\ ESTHER TORRADO MARTÍN-PALOMINO \\ Instituto Universitario de Estudios de las Mujeres, Universidad de la Laguna
}

Recibido: $24 / 03 / 2014$

Aceptado: 12/05/2014

\section{Resumen}

Los valores relacionados con la familia y la estructura familiar son decisivos para consolidar las trayectorias profesionales de las mujeres científicas. Al mismo tiempo, las condiciones laborales y profesionales en ciencia (movilidad, implicación en el trabajo, etc.) condicionan las estructuras familiares de estas mujeres. Los resultados de este trabajo revelan que las investigadoras españolas muestran un fuerte compromiso con la familia pero también con sus aspiraciones profesionales. Para lograr ambos objetivos, las mujeres desarrollan diferentes estrategias familiares que son analizadas en este trabajo.

Palabras clave: Biografías de vida, carreras científicas, familias con carreras paralelas, parejas colaboradoras, parejas mentoras, parejas adversarias, maternidad, profesión, ciclo de vida

\begin{abstract}
Values related to family and family structure are decisive to the professional progression of women scientists. At the same time, professional and labour conditions in science (mobility, working intensity, etc.) influence family structures of these women. The results of this work show that Spanish researchers display a strong commitment to the family and also their professional aspirations. To achieve both goals, women develop different family strategies which are analysed in this work.
\end{abstract}


Keywords: Biographies, scientific careers, dual career households, collaborative partners, mentoring partners, adversary partners, maternity, profession, life course. 


\section{Introducción}

Los estudios feministas han dedicado un gran esfuerzo a describir la situación de las mujeres en las instituciones académicas y científicas. A partir de estos estudios conocemos el incremento de mujeres investigadoras y su distribución desigual entre los distintos campos del conocimiento, puesto que siguen siendo minoritarias en las áreas técnicas y de ingenierías. También sabemos que mientras el $45 \%$ de las mujeres ocupan posiciones de ayudante o becaria, aproximadamente el $15 \%$ son titulares, catedráticas o profesoras de investigación ${ }^{1}$. Este fenómeno denominado suelo pegajoso suele achacarse a la maternidad; puesto que las mujeres son las que tienen hijos y, generalmente, dedican más tiempo a la familia, lo cual parece ralentizar sus carreras. Pero ello suscita una pregunta inmediata, ¿qué estrategia utilizan las catedráticas y profesoras de investigación que tienen hijos y lo logran? En realidad, conocemos sólo muy superficialmente las estrategias familiares de las mujeres científicas. Desconocemos cómo organizan su tiempo², o si seguir el ritmo altamente competitivo de una carrera profesional requiere cambios en las estructuras familiares de estas mujeres.

Nos proponemos analizar las estrategias familiares que utilizan las mujeres científicas para incorporarse, promocionarse y alcanzar posiciones de responsabilidad en las instituciones académicas o de investigación. Ello nos permitirá conocer mejor las barreras a las que se enfrentan y las oportunidades que encuentran, en relación a la familia (los hijos y las parejas). Situaremos a las investigadoras en su contexto familiar y laboral, como agentes fundamentales

1. Datos del 2007 del informe: UNIDAD DE MUJERES Y CIENCIA: Libro Blanco. Situación de las Mujeres en la Ciencia Española, Madrid, Ministerio de Ciencia e Innovación, 2011. También pueden encontrarse resultados similares en: GARCíA DE CORTÁZAR, M. Luisa et al.: Mujeres y hombres en la ciencia española. Una investigación empírica, Madrid: Instituto de la Mujer, 2006; GARCÍA DE LEÓN, M. Antonia et al.: Las académicas: profesorado universitario y género, Madrid: Instituto de la Mujer, 2001; PÉREz SEDEÑo, Eulalia (ed): Las mujeres en el Sistema de Ciencia y Tecnología, Estudios de casos, OEI, 2001.

2. LYON, Dawn y WOODWARD, Alison E. «Gender and Time at the Top: Cultural Construction of Time in High-Level Careers and Homes», European Journal of Women's Studies, 11, 2, 205-221, 2004. 
de su entorno, es decir, con capacidad para gestionarlo. Examinaremos si su gestión del entorno está siendo transformadora o reproductora de los roles tradicionales de género. Y, abordaremos los posibles cambios que se pueden estar produciendo en las estructuras familiares de las mujeres investigadoras. Contamos además con protagonistas muy relevantes para analizar las posibilidades de cambio puesto que son personas altamente cualificadas, de quienes podemos asumir a priori que tienen más recursos para gestionar las relaciones de género en términos de igualdad.

Las trayectorias de las mujeres incluidas en este estudio están encuadradas en culturas institucionales y contextos laborales que definen sus oportunidades profesionales. Pero también dentro de un contexto social y de valores sobre la familia y los roles de género que condiciona las expectativas y las estructuras familiares. Por ello tendremos en cuenta elementos claves sobre la familia, la situación socio-económica, la pareja, la situación de movilidad tan característica de la población investigadora y la red de cuidados formal e informal de los hijos.

Adoptaremos una perspectiva metodológica basada en el ciclo de vida, lo cual implica conocer cómo se han desarrollado las trayectorias de las mujeres investigadoras a lo largo del tiempo. Analizaremos la influencia de la familia en sus carreras profesionales, y la del contexto laboral en la estructura familiar (relacionada, por ejemplo, con la maternidad, el divorcio o la separación, etc.), que se produce a lo largo de las distintas etapas de sus vidas. En definitiva, tendremos en cuenta que las familias y los roles de género cambian a lo largo del ciclo vital a medida que se van consolidando posiciones laborales y los hijos se hacen mayores.

Finalmente, este trabajo también pretende llenar un vacío en los estudios de género en España ${ }^{3}$ centrado en el análisis de las biografías de mujeres científicas, abordando su entorno familiar. Por tanto, este trabajo pretende realizar un primer análisis de las estructuras y estrategias familiares de las mujeres investigadoras ${ }^{4}$.

3. Pycior, Helena, M., G. Slack, Nancy y AbiR-Am, Pnina G. ed.: Creative Couples in the Sciences, Rutgers, 1996; Monnosson, Emily ed.: Motherhood, the elephant in the laboratory, Cornell University Press, 2008; SCHIEBINGER, Londa, DAVIS HENDERSON, Andrea y Gilmartin, Shannon K.: Dual-Career Academic Couples. What Universities Need to Know, Michelle R. Clayman Institute for Gender Research at Stanford University, Stanford, CA, 2008.

4. Aunque esta línea de investigación no se ha desarrollado suficientemente en España, sí contamos con buenos ejemplos de estudios biográficos sobre sujetos históricos: CABRÉ, Monstserrat: «La Ciencia de las mujeres en la Edad Media. Reflexiones sobre la autoría femenina», en C. SEGURA Graiño (ed.): La voz del silencio II, Madrid, Almudayna, 


\section{La dialéctica entre la familia y la profesión científica}

Familia y carrera profesional parecen oponerse. La constatación de que las mujeres tienen más dificultades para promocionarse y acceder a puestos de responsabilidad al mismo ritmo que sus compañeros varones suele plantearse como una cuestión de elección de las mujeres que priorizan la maternidad. El argumento más usado por la comunidad científica es que desde el momento en que las mujeres forman un núcleo familiar y tienen hijos, su productividad científica disminuye. El estudio longitudinal de Xie y Shauman ${ }^{5}$ muestra que las mujeres casadas y con hijos presentan una proporción significativamente más desventajosa que sus compañeros a la hora de promocionarse académicamente.

Los estudios realizados en España también sugieren que los hijos son un obstáculo en las carreras de las mujeres. Por una parte, Barral et $\mathrm{al}^{6}$ en un estudio sobre una muestra de nueve científicas muy notables constatan que suelen tener pocos hijos y que las medidas de apoyo (escuelas, redes de cuidado infantil, etc.) han sido fundamentales para conseguir los logros alcanzados. Por otra parte, la Unidad de Mujeres y $\mathrm{Ciencia}^{7}$, en relación a los datos de recursos humanos en ciencia y tecnología del INE, apunta que las mujeres con hijos menores de 18 años publican una proporción media de 1.1 artículos menos que los hombres con hijos menores $(-1.96,+0.86)$. Se evidencia la misma tendencia respecto a la publicación de libros, dirección de tesinas y tesis, por lo que las autoras concluyen que la maternidad penaliza los resultados de las mujeres. Así, un 38\% de las catedráticas tienen hijos frente al 63\% de catedráticos y, paralelamente, un $21 \%$ de las catedráticas son solteras frente a un $15 \%$ de catedráticos solteros. Similares datos ya habían sido establecidos por García de Cortázar ${ }^{8}$, en el estudio de 2006 Mujeres y Hombres en la Ciencia

1993; OrTíz Gómez, Teresa y BeCerra Conde, Gloria (eds.): Mujeres de ciencia. Mujer, feminismo y ciencias naturales, experimentales y tecnológicas, Granada, Universidad de Granada, 1996; MAgallón, Carmen: Pioneras españolas en las ciencias. Las mujeres del Instituto Nacional de Física y Química, Madrid, CSIC, 1998; SANTESMASES, M. Jesús: Mujeres científicas en España (1940-1970). Profesionalización y modernización social, Madrid, Instituto de la Mujer, Ministerio de Trabajo y Asuntos Sociales, 2000; BARRAL, María José, Delgado, Isabel, TuRRado, Teresa y Magallón, Carmen: «Life Paths of Successful Women Scientists in Spain», Géneros, Multidisciplinary Journal of Gender Studies, 3 (1): 351-372.

5. Xie, Yie y Shauman, Kimberlee A.: Women in Science: Career Processes and Outcomes, Harvard University Press, Cambridge, Massachusetts, 2003.

6. BARRAL, María José et al., op. cit.

7. UNIDAD DE MUJERES Y CIENCIA, op. cit.

8. García de Cortázar, M. Antonia, op. cit. 
donde se establecía que el $20 \%$ de las mujeres científicas eran solteras, en cambio, sólo $8 \%$ de los hombres científicos eran solteros.

Sin embargo, el trabajo de Mary F. Fox ${ }^{9}$ sugiere que la relación entre familia, género y productividad científica es mucho más compleja. Según esta autora, el nivel de productividad científica varía dependiendo del tipo de familia, puesto que las mujeres casadas en segundas nupcias presentan ratios más altos que las mujeres casadas por primera vez. En relación a los hijos, Fox sostiene que la productividad de las mujeres con hijos en edad preescolar es mayor que entre aquellas que no tienen hijos o que tienen hijos en edad escolar. Por último, la autora establece una relación positiva entre productividad científica y el hecho de que las mujeres estén casadas con otros científicos (aspecto al cual le dedicaremos mayor atención más adelante).

Matrimonio y ciencia también han mantenido una relación ambivalente históricamente. Algunas mujeres fueron pioneras en campos científicos emergentes de la geología, la botánica, la ecología o la astronomía al ayudar a sus parejas ${ }^{10}$. Etzkowitz plantea que las mujeres aparecen en campos innovadores de la ciencia cuando éstos no son relevantes para los hombres pero, más tarde, cuando estos campos tienen una relevancia dentro del ámbito científico, las mujeres desaparecen ${ }^{11}$. Por tanto, la invisibilización de las mujeres en la actividad científica parece estar más relacionada con el prestigio de la actividad científica que con las cargas familiares de las mujeres. Por otra parte, los estudios históricos también ponen de manifiesto que las mujeres son relegadas a una posición de subordinación no sólo en la ciencia sino también en sus estructuras familiares, ya que son sus padres y parejas quienes las invisibilizan. La publicación de los resultados con nombres masculinos, para no infringir las normas sociales, hacía que el trabajo de estas mujeres quedara relegado a favor de la autoría de sus padres, maridos y parejas que recibían todo el crédito ${ }^{12}$.

9. Fox, Mary F: «Gender, Family Characteristics, and Publication Productivity among Scientists», Social Studies of Science, 35 (1), 131-150, 2005.

10. PYCIOR, Helena et al., op. cit.

11. ETZKowitz, Henry y RANGA, Marina: «Gender dynamics in science and technology: From the "Leaky Pipeline" to the "Vanish Box"», Brussels Economic Review, Special Issue «Beyond the leaky pipeline - Challenges for research on gender and science», 54 (2/3): 131-148, Summer-Autumn 2011.

12. PYCIOR, Helena et al., op. cit., Rossiter, Margaret W.: «The Matthew Matilde Effect in Science», Social Studies of Science, vol. 23 (2): 325-341, 1993; MiqueO, C. et al.: «Del análisis critico a la autoridad femenina en la ciencia, Feminismo/s, 1, 195-216, junio 2003. 
La relación entre familia y ciencia parece mucho más compleja que la atribución de que los hijos y las elecciones familiares suponen el lastre más importante para las carreras profesionales de las mujeres. En primer lugar, hay un contexto histórico y generacional que es importante tener en cuenta porque condiciona la concepción sobre familia y carrera profesional, los roles de colaboración y compromiso dentro de la familia y las relaciones de género. Estos modelos estarían siendo modificados total o parcialmente en la actualidad por las familias, en particular, las mujeres. Por ejemplo, algunas mujeres tienen un estado civil distinto al de casadas por propia elección, y la maternidad no es el único hito vital que podrían perseguir. Las mujeres científicas suelen presentar mayores porcentajes de soltería y divorcio, y suelen tener menor número de hijos ${ }^{13}$. También es posible otro equilibrio de poderes dentro de la estructura familiar. Por ejemplo, los estudios sobre movilidad internacional destacan que las mujeres pueden ser las que lideren el proyecto familiar migratorio y, por tanto, la familia acepta cambiar de residencia porque la madre y no el padre es quien tiene mejores expectativas profesionales y/o salariales ${ }^{14}$.

Sin embargo, el empoderamiento de las mujeres, debido a una situación laboral y económica ventajosa, no es suficiente para asegurar una distribución de roles diferentes dentro del núcleo familiar. Las mujeres aún siguen siendo las responsables principales de la organización familiar y, a pesar de que haya mayor colaboración entre las parejas y miembros de la familia, suelen ser quienes planifican y controlan la ejecución de las tareas ${ }^{15}$. Por tanto, tener una actitud progresista y una pareja colaboradora no es un elemento transformativo por sí solo, pues las actitudes respecto al cuidado de la familia y los hijos siguen vinculadas prioritariamente a las mujeres.

El hecho de que un alto porcentaje de mujeres investigadoras establezcan relaciones sentimentales con compañeros científicos sugiere que con ellos es

13. XIE y Shauman, op. cit., Kulu, Hill: «Migration and Fertility: Competing Hypotheses Re-examined», European Journal of Population, 21, 51-87, 2005.

14. GREEN, Andy E.: "A question of compromise? Case study evidence on the location and mobility strategies of dual career households», Regional Studies, 31, 7, 641-57, 1997; GONZÁLEZ RAMOS, Ana M. y VERGÉS BOSCH, Núria: «International mobility of women in S\&T careers: Shaping plans for personal and professional purposes», Gender, Place and Culture, 20, 5, 613-629, 2013; EVERGETI, Venettia y RYAN, Louise: «Negotiating transnational caring practices among migrant families». En KRALER, Albert et al. (eds.) Gender, Generations and the Family in International Migration. Utrech: Amsterdam University Press, 2011.

15. HerTz, Rosanna: More Equal Than Others: Women and Men in Dual-Career Marriages, University of California Press, Berkeley, CA, 1986; HochsCHILD, Arlie y MACHUNG, Anne: The Second Shift, New York: Penguin, 1989. 
más fácil gestionar los intereses de hombres y mujeres en el ámbito profesional y familiar ${ }^{16}$. Constituir una familia con parejas que desarrollan carreras paralelas puede proporcionar un contexto de complicidad y compromiso para las mujeres. No sólo comparten una visión común, sus planes profesionales y personales también se acoplan en el tiempo, es decir, a lo largo del ciclo vital. La formación de una familia y las distintas etapas de la carrera profesional se ajustan para ambos miembros de la pareja. Algunos estudios ${ }^{17}$ han destacado que las mujeres consideran que son incomprendidas cuando trabajan con parejas que pertenecen a otras áreas: no entienden el ritmo de trabajo, la intensificación de la actividad, las horas dedicadas al trabajo en los momentos de descanso, etc. Por eso, como destaca el estudio de Schiebinger ${ }^{18}$, las mujeres tienden a estar comprometidas con científicos en mayor medida que los hombres con científicas. Quizá los hombres tienen más oportunidades de tener una pareja no científica que las mujeres o las mujeres dependen en mayor medida que los hombres de ser comprendidas por sus parejas.

La administración de los tiempos y la conciliación de la vida familiar y profesional dependen también del nivel socio-económico de la familia ${ }^{19}$. La posición de clase supone un punto de partida nada desdeñable para las mujeres investigadoras. Incluso cuando esa posición no es por adscripción sino adquirida, disfrutar de un mejor nivel socio-económico es un elemento fundamental, por ejemplo, para conciliar trabajo y familia. En cambio, la inestabilidad en la ciencia y los bajos niveles salariales redunda en una mayor dependencia de los servicios públicos, la red de cuidado, la educación de los niños o el cuidado informal de parientes y amigos. En definitiva, los recursos institucionales (colegios, guarderías, medidas de flexibilidad laboral, horarios reducidos y permisos de maternidad entre otros) cumplen una función social para las mujeres investigadoras. Particularmente para aquellas que, como suele ocurrir en el ámbito científico, residen temporalmente en otros países. El trabajo realizado por Vergés y González ${ }^{20}$ sobre mujeres que presentan una

16. SCHIEBINGER, Londa et al., op. cit.

17. González Ramos, Ana M. y Vergés Bosch, Núria, op. cit.

18. SCHIEBINGER, Londa, op. cit., p. 14.

19. GonZÁlez RAmos, Ana M.: «Victoria y la ciencia: El papel de las mujeres en la ciencia contemporánea» en CASTAÑO, Cecilia (dir.): Mujeres Construyendo la Sociedad del Conocimiento: Ciencia, Tecnología y Género, Barcelona: Editorial Aresta, 2014.

20. Vergés Bosch, Núria y GonZÁlez Ramos, Ana M.: «Beyond the Work-Life Balance: Family and International Mobility of the Highly Silled», Sociología y Tecnociencia. Revista Digital de Sociología del Sistema Tecnocientífico, 3, 55-76, 2013. Sin embargo la tesis contraria, es decir, que las parejas presentan probabilidades más altas de romperse si se mueven frecuentemente es sostenida por BOYLE, Paul J. et al (ed.) «Moving and union dissolution», Demography, 45, 1, 209-222, February 2008. 
alta tasa de movilidad internacional sugiere que la experiencia de vivir sin parientes acentúa el sentimiento de unidad familiar y fortalece los sentimientos entre los miembros de la familia.

\section{Metodología de análisis para abordar las estrategias familiares y las trayectorias de las mujeres investigadoras}

El presente estudio analiza las estrategias profesionales desarrolladas por 28 mujeres científicas y tecnólogas contemporáneas. Sus perfiles corresponden a diferentes cohortes de edad, áreas de conocimiento y trayectorias profesionales. A través del relato de sus vidas y sus discursos indagamos sobre los modelos de familia y las posibles transformaciones de género que se producen en sus estructuras familiares. Para ello tenemos en cuenta factores como la edad, el contexto socio-económico y laboral, el entorno institucional ligado a su área de conocimiento y las actitudes sobre la familia, los hijos y las relaciones de pareja. Establecemos comparaciones entre los modelos de familia descritos por las investigadoras y sus logros profesionales; particularmente, asociados a cambios significativos que se hayan producido a lo largo del ciclo vital en sus familias (hijos, divorcios, etc.).

La elección de las mujeres entrevistadas sigue un método no aleatorio resultante de la combinación de diferentes metodologías. Se utilizaron estrategias de selección por conveniencia, bola de nieve y muestreo intencionado destinados a cumplir los objetivos planteados. Estos objetivos estaban relacionados con la necesidad de encontrar diversidad en nuestra población. Por tanto, teniendo en cuenta tres dimensiones fundamentales: la procedencia geográfica (si son extranjeras residentes o nacidas en España), las áreas de conocimiento y la pertenencia a cohortes generacionales diferentes. Conocer mejor las características que rodean a las mujeres permite comprender mejor los resultados hallados. Por esta razón, a continuación, describiremos brevemente a las investigadoras, que guardan su anonimato.

Puesto que el objetivo de este estudio se centra en el sistema español de ciencia y tecnología, la mayoría de las mujeres que conforman la muestra son españolas. Aún así, se consideró oportuno incluir a cinco extranjeras con una larga trayectoria en España, lo cual aporta un punto de vista comparativo transnacional. Nuestra hipótesis de partida es que sus opiniones diferirían de las españolas debido a características culturales o quizá a su estatus de extranjeras. La movilidad de las investigadoras también ha ofrecido resultados interesantes sobre cambios familiares en este grupo de mujeres.

De acuerdo a la segunda dimensión, las áreas de conocimiento, siete mujeres pertenecen a diversas ramas de las ingenierías, seis a las ramas 
físico-naturales, seis a las ciencias sociales y humanas, cinco a ciencias de la vida, y cuatro a ramas de biología y ciencias de la naturaleza. La distribución entre áreas de conocimiento está intencionadamente sesgada hacia áreas donde las mujeres son tradicionalmente minoritarias ${ }^{21}$ puesto que preveíamos que esas investigadoras habrían experimentado más obstáculos en sus trayectorias profesionales.

Finalmente, la tercera dimensión está dirigida a comparar estrategias de investigadoras pertenecientes a distintas generaciones. Cuatro mujeres se sitúan alrededor de los 35 años de edad; el grupo más numeroso lo constituye quince mujeres entre 40-60 años; y, finalmente, tres mujeres tienen más de 60 años. La distribución por edades corresponde con una estrategia de selección destinada a asegurar la presencia de mujeres en tres las etapas de la carrera investigadora ${ }^{22}$. Las mujeres seleccionadas en el primer grupo tienen el doctorado finalizado o están muy cerca de alcanzarlo; al mismo tiempo tienen una trayectoria suficientemente amplia para evaluar sus primeros logros y desaciertos. El segundo grupo está constituido por mujeres sénior con una línea de investigación propia y posición permanente en el sistema de ciencia y tecnología. Finalmente, el tercer grupo de mujeres ya han completado su trayectoria profesional, están jubiladas o muy próximas a ello. Algunas de estas mujeres habían sido pioneras en sus áreas de conocimiento, por lo que hemos obtenido un relato muy valioso sobre los primeros momentos de la ciencia española relacionados con el acceso de las primeras mujeres.

Mientras las mujeres pertenecientes a las ciencias sociales se distribuyen más homogéneamente entre las tres cohortes de edad, las mujeres de edad más sénior están más representadas en las ramas de ciencias de la vida y las júnior en las ramas de ingeniería o tecnológicas.

Las narrativas de sus trayectorias de vida fueron obtenidas mediante entrevistas en profundidad que duraron aproximadamente hora y media o dos horas, aunque algunas fueron grabadas en dos sesiones y se alargaron en el tiempo. La estructura de la entrevista adoptó un modelo de preguntas abiertas a través del cual se desarrollaron las siguientes dimensiones de análisis:

- Estrategias y acontecimientos que facilitaron el desarrollo de sus carreras profesionales.

21. Pérez Sedeño, Eulalia, op. cit., García de León, M. Antonia et al. Op. Cit. García de CortázAr, M. Luisa et al. Op. Cit., UMYC, Op. Cit.

22. O'Neil, Deborah A. y BilimoriA, Diana «Women's career development phases. Idealism, endurance, and reinvention, Career Development International, 10, 3, 168-189, 2005. 
- Concepción de la familia, las parejas y los hijos en relación al ejercicio de la profesión.

- Influencia de la pareja en su trayectoria profesional.

- Modos de conciliación de la vida familiar, profesional y personal, donde se incluye el cuidado de los hijos y de las personas dependientes y también el descanso, el ocio o el tiempo libre.

- Obstáculos institucionales o que provienen de una valoración social sesgada del rol de las mujeres.

Por último, queremos hacer hincapié en un rasgo pertinente del diseño de este estudio. Si bien, los estudios biográficos históricos suelen elegir perfiles de investigadoras exitosas, nosotras decidimos incluir tanto a mujeres que habían logrado sus objetivos profesionales (es decir, habían obtenido un reconocimiento explícito de la comunidad científica), como a aquellas que no los habían logrado (es decir, abandonaron o no consiguieron avanzar al nivel esperado dentro de sus expectativas). De hecho, la mayoría de las mujeres no pertenecen a ninguno de estos dos grupos de una manera clara, ya que siguen trabajando para alcanzar mayores logros. Aún así, este estudio incluye dos mujeres que podríamos catalogar como ejemplos de fracaso pues abandonaron su carrera científica, y otras mujeres como exitosas porque acumulan premios y son respetadas por sus logros científicos.

\section{Estrategias de gestión de la familia y la profesión}

Como indica el título de este apartado, los resultados de nuestro estudio sugieren que las mujeres han de gestionar una estrategia familiar que se adecúe a sus objetivos profesionales y a sus propias estructuras familiares, al mismo tiempo que a sus contextos laborales y económicos. Además, estas circunstancias cambian a lo largo de la vida, por lo que también cambian las estrategias, los modelos de familia y los roles de género. Hemos organizado este apartado de resultados en torno a tres aspectos claves: la concepción de la familia y el trabajo, el rol de las parejas y la gestión de la cotidianidad de la familia.

\subsection{Profesión + familia}

La maternidad y la familia juegan un papel muy importante en las trayectorias de las investigadoras incluidas en el estudio. La mayoría de ellas tienen hijos aunque no más de dos. Todas eligieron el momento idóneo para concebirlos, normalmente después de haber conseguido una posición permanente, de ellas mismas o de sus parejas. En los primeros momentos, especialmente si la persona que obtiene la plaza es la pareja, las carreras femeninas se ralentizan. 
La recuperación del ritmo depende de muchos factores azarosos y también estructurales, por ejemplo, en aquellas áreas más masculinizadas y técnicas, más ligadas al trabajo en laboratorios y en equipo, es más difícil mantener el mismo nivel de productividad que sus compañeros varones si no tienen el apoyo de sus compañeros ${ }^{23}$. La mayoría de las mujeres sostienen que los hijos influyen en los logros profesionales, de lo cual se deduce que mantengan una actitud muy precisa sobre la planificación de la maternidad y auto-organización respecto al cuidado del hogar y los hijos.

La excepción la presenta una de las investigadoras de nuestro estudio que decidió tener su hija a pesar de la situación precaria en que se encontraban tanto ella como su pareja. En sus carreras profesionales se habían producido muchas circunstancias azarosas y planificadas, habían desarrollado carreras interrumpidas con numerosas estancias en diversos países que no les habían permitido incorporarse al sistema de ciencia español, con un efecto negativo. Esa situación lastimosa fue para ellos el detonante para elegir una estrategia alternativa que pasaba por centrarse en la familia, y que ellos valoraban como una mejora sustancial en sus vidas. Finalmente, ambos han logrado un empleo estable aunque en ciudades algo distantes, lo cual conlleva problemas importantes de conciliación, asunto que trataremos en un siguiente apartado.

Sólo unas pocas mujeres han decidido no tener hijos. En concreto, nueve informantes de las que se encuentran entre 40-55 años de edad (para valorar esta cuestión prescindimos del grupo de edad más joven porque podrían tenerlos en el futuro). Para algunas, la decisión no fue consciente sino el resultado de su trayectoria; preocupadas por alcanzar logros profesionales $\mathrm{y}$, por tanto, la maternidad era un asunto que podía esperar. Ninguna de estas mujeres pone como excusa su profesión para justificar no tener hijos. Otro caso distinto es el de una catedrática de un área altamente masculinizada, con dificultades de fertilidad, que finalmente optó por la adopción. La decisión la tomó después de obtener la plaza, lo que ella interpreta como una ventaja profesional respecto a sus compañeras en el área. Para un grupo minoritario de mujeres, tener hijos supone una carga social que imposibilita cumplir con sus expectativas profesionales. Por ejemplo, una investigadora del área de ciencias de la vida explica que había tomado esa decisión antes de incorporarse a la investigación, como resultado de su experiencia familiar, ya que las mujeres de su familia siempre cumplieron un rol tradicional con el cual se sentía disconforme. Concretamente, habla de la situación de su hermana

23. BAgilhole, Barbara y Goode, Jackie «The Contradiction of the Myth of Individual Merit, and the Reality of a Patriarchal Support System in Academic Careers: A Feminist Investigation, European Journal of Women's Studies», 8, 2, 161-180, 2001. 
mayor que había abandonado sus aspiraciones profesionales para cuidar de su propia familia.

Por tanto, la familia es un factor predominante para la mayoría de las investigadoras de este estudio que, sin embargo, no tiene una única repercusión negativa sobre sus expectativas profesionales. Depende de su actitud sobre la familia y la profesión. Una pedagoga representa una estrategia orientada hacia la familia quien, al haber seguido a su marido al extranjero, abandonó su carrera profesional para dedicarse al cuidado de sus dos hijos. Éste podría ser un ejemplo de abandono, pero ella insiste en que es una situación temporal y que tras la crianza de sus hijos volverá a reanudar su trayectoria profesional. El otro caso de abandono profesional es el de una astrofísica que actualmente tiene una hija, pero su decisión de dejar la carrera profesional no se relaciona con la maternidad. Las razones que la impulsaron a abandonar la carrera profesional tras haber realizado el posdoc fue su desilusión por la manera en que se regula la ciencia y la academia. De hecho, su hija nació cinco años después de que abandona la astrofísica, y sólo después del establecimiento de una nueva relación; simplemente, el modelo de éxito y de acumulación de méritos no se adecuaba a su escala de valores.

Otro aspecto interesante sobre la maternidad es que las mujeres con hijos siguen desarrollando su carrera profesional a pesar de estar divorciadas o residir en países diferentes al de sus parejas, representando un modelo de familia monoparental al menos temporalmente. Un ejemplo de este perfil es una investigadora rusa que ha estado separada de su marido y su hijo mayor durante quince años, mientras la acompañaba su hija más pequeña por los distintos países europeos donde residió debido a su precaria salud y por razones de apego. La movilidad no es un inconveniente para las mujeres solas con hijos a pesar de que la mayoría de las mujeres tienen un discurso contrario, es decir, piensan que la maternidad dificulta la movilidad internacional. Aún así, se mueven con sus hijos menores de edad cuando las circunstancias así lo requieren.

Por tanto, destacamos de las mujeres integrantes de este estudio su gran compromiso con la investigación y con sus familias. Su comportamiento demuestra una gran determinación y sentido de la planificación. Su tenacidad y resolución son características frecuentes, tanto para las mujeres que han logrado ciertos éxitos como para las que no han obtenido resultados tan satisfactorios. Seguramente estos rasgos de personalidad han sido decisivos para lograr una estrategia de auto-inclusión en la ciencia, a la vez compatible con sus parejas y sus familias. 


\subsection{Parejas Científicas: Colaboradoras y adversarias}

El mundo académico ${ }^{24}$ ha descrito distintos perfiles para las parejas compuestas por dos profesionales que comparten intereses y entornos laborales comunes. Las relaciones que mantienen entre ellos pueden ser de colaboración o de oposición. Son colaboradoras cuando las parejas se complementan y apoyan mutuamente, tal y como sucede cuando desarrollan carreras paralelas o cuando uno de ellos ejerce de mentor del otro. Por el contrario, las parejas pueden ejercer un efecto negativo en la trayectoria profesional o en su vida cuando se presentan como adversarios, es decir, compañeros que compiten por los mismos recursos, invisibilizan los logros de su pareja u obstaculizan su desarrollo profesional. Estas categorías no son puras ni estables en el tiempo, pues pueden cambiar a lo largo de la carrera profesional y del ciclo vital.

En este estudio, las investigadoras que tienen compañeros científicos son mayoría, tal y como afirma Schiebinger ${ }^{25}$. Trece de las diecinueve parejas con carreras paralelas en el estudio comparten además la misma área de conocimiento. De ellas, diez parejas mantienen cierto nivel de colaboración conjunta en sus líneas de trabajo. En el caso de las mujeres de mayor edad, las mujeres suelen agradecen a sus maridos el apoyo recibido en las etapas iniciales de su carrera. Afirman que sus parejas les permitieron incorporarse a la actividad científica o les facilitaron alcanzar logros profesionales. Una mujer explica que casarse con él fue un pasaporte para viajar juntos a Estados Unidos durante la época franquista, puesto que las normas sociales eran mucho más estrictas de lo que son ahora y habría sido imposible para ella salir sola. Después, él le cedió la línea de investigación que habían estado desarrollando durante los primeros años de su carrera, y que sería decisiva para su reconocimiento internacional, mientras él ponía en marcha otra línea de investigación menos relevante dentro del área de conocimiento. Otra pareja supone un ejemplo de cambio de roles dentro de la «familia científica ${ }^{26}$. En las primeras etapas de la carrera profesional, él apoyaba la carrera de su mujer porque desarrollaban una línea muy productiva científicamente, en el área de las ciencias agrícolas, en un ambiente muy masculinizado y poco propicio al desarrollo de carreras femeninas. Casi en la edad de la jubilación de su marido, es ella quien lo acoge

24. Pycior, Helena et al., op. cit. Monosson, Emily ed., op. cit. HerTz, Rosanna, op. cit. WAGNER, Izabela «Career Coupling: Career Making in the Elite World of Musicians and Scientists, Qualitative Sociology Review, II, 3, 78-98, diciembre 2006.

25. SCHIEBINGER Londa et al., op. cit.

26. Este término ha sido acuñado para describir parejas de científicas unidas por un trabajo científico común como la familia Curie, PYCIOR, Helena et al., op. cit. 
a él en su equipo de investigación, donde ella lidera una línea muy innovadora y exitosa diferente a la que lideraba él.

La relación de mentorazgo es una de las relaciones más comunes entre las parejas científicas de este estudio. El rol de mentor suele estar protagonizado por un hombre que tiene más edad o, bien, experiencia profesional. En algunos casos, ellos han sido profesores de ellas; en otros, la relación es más igualitaria, son compañeros en un campo del conocimiento al que ella ha llegado posteriormente; o, también, aunque no pertenezcan a la misma área de conocimiento, el mentor tiene más experiencia y conoce mejor el funcionamiento y las normas del mundo académico y de investigación.

En otras ocasiones la relación de colaboración es una forma de apoyo mutuo, de colaboración igualitaria e incluso de superación de las barreras profesionales que comparten. Así, una pareja sénior consiguió financiación para un laboratorio donde pusieron en marcha sus experimentos; de esta manera, escapaban de la falta de estímulos y el escaso desarrollo de la neurociencia española en los años 70. Como otras muchas parejas donde ambos tienen la misma edad, han seguido una progresión profesional paralela y no han tenido hijos. Empezaron a publicar al mismo tiempo, compaginaron su periodo de posdoc en el mismo país, y volvieron a España juntos donde en la actualidad dirigen un grupo de investigación. Sin embargo, él recibe mayor reconocimiento que ella teniendo los mismos méritos profesionales.

También hay casos de ralentización de las carreras femeninas, especialmente, cuando se apuesta por la familia o por la carrera del compañero. Esa decisión está influida por la imagen de éxito que representa el compañero o porque su posición conlleva una situación económica más ventajosa para la familia. Es el caso de algunas mujeres que siguieron a sus compañeros hasta el extranjero porque las expectativas de sus compañeros eran mejores, y a pesar de que esa situación no les beneficiaba particularmente. La decisión de marchar se toma después de una negociación por parte de la pareja ${ }^{27}$. Otro ejemplo típico de ralentización de las trayectorias femeninas se produce cuando las mujeres cambian de sector productivo (normalmente del privado al público) o aceptan una jornada a tiempo parcial al nacer los hijos.

En cuanto a las parejas realmente adversarias, es decir, que han impedido que sus compañeras realizaran sus aspiraciones profesionales, en el estudio encontramos una mujer de más de 60 años que describe a su marido como una buena persona pero machista. Él fue un obstáculo para que ella desarrollase una carrera en el campo de la salud infantil, como había aspirado

27. González Ramos, Ana M. y Vergés Bosch, Núria, op. Cit. 
durante la primera etapa de su carrera profesional. Aún así y a pesar de las barreras que se encontró en la España franquista y en su estructura familiar (ser madre de una familia numerosa), en su madurez consiguió el título de doctor en medicina. Entre las parejas más jóvenes, este tipo de parejas no se visibilizan. Una razón es que las investigadoras hablan de ex-parejas con las que no se llevaban bien o con las que dejaron de compartir intereses y "planes de vida», pero no expresan explícitamente que se oponían a sus aspiraciones profesionales. Por tanto, podemos concluir que, para las mujeres investigadoras contemporáneas, el divorcio puede ser una manera de superar una pareja adversaria.

\subsection{Estrategias familiares y de conciliación}

Lyon y Woodward ${ }^{28}$ sugieren que la asimetría de género en la sociedad favorece que los hombres sigan movilizando el altruismo de las mujeres para hacerse cargo del hogar y del cuidado familiar. Esta circunstancia es, sin duda, un escollo para la progresión de las mujeres en ciencia. Las políticas públicas han sido la respuesta tradicional que se ha ofrecido a las mujeres profesionales. Cuanto mayor nivel de recursos institucionales tenga una sociedad, más oportunidades tendrán las mujeres de desarrollar sus carreras científicas ${ }^{29}$. Sin embargo, las políticas de apoyo a la conciliación no parecen ser suficientes, puesto que muchas mujeres encuentran dificultades para promocionarse y ocupar posiciones de responsabilidad en ciencia.

La posición socio-económica mitiga los efectos negativos de la conciliación familiar o los acentúa, de acuerdo a las trayectorias profesionales de las investigadoras de este estudio. Las mujeres de clase alta declaran abiertamente que la crianza de los hijos y el cuidado del hogar no han supuesto nunca un problema adicional para sus carreras profesionales, pues contaron con personas contratadas a tiempo completo que asumieron estas tareas. En cambio, representa un problema para la mayoría de las mujeres del estudio, incluso las de clase media, y afecta muy especialmente a aquellas que sufren precariedad laboral y salarial. Además, la mayoría de las mujeres científicas no cuentan con familiares directos que puedan hacerse cargo de sus hijos pequeños porque viven en otras ciudades y, temporalmente, también en otros países. Por

28. Lyon, Dawn y WoOdWARd, Alison E., op. cit.

29. Etzkowitz Henry, Kemelgor, Carol y Uzzi, Brian: Athena Unbound: The Advancement of Women in Science and Technology, Cambridge University Press, Cambridge, 2000; NAS Committee on Science, Engineering and Public Policy: Beyond bias and barriers: Fulfilling the potential of women in academic science and engineering, Washington, D.C., National Academies Press, 2007. 
tanto, la conciliación se hace a través de la red de servicios públicos (escuelas, guarderías) y del compromiso de los dos miembros de la pareja.

El ajuste necesario entre la red de recursos públicos y la flexibilidad laboral es decisivo para conciliar obligaciones profesionales y laborales y, en definitiva, para gestionar la cotidianidad. Las dificultades añadidas, como las relatadas por la pareja citada anteriormente con posiciones estables en universidades ubicadas en distintas ciudades, dificulta enormemente la conciliación. No sólo es la carrera profesional la que se resiente sino el tiempo de ocio y descanso: si aumentan las horas diarias empleadas en desplazamiento, disminuye el tiempo útil para llevar a cabo tareas cotidianas pero esenciales. Todas estas tareas se acaban cumpliendo, sacrificando el tiempo de ocio y de descanso. Por eso, cuando se pregunta a las parejas que viven en el extranjero o que son extranjeras cómo es la vida sin abuelos y familiares, ellos destacan que se agradece que vengan de visita porque así se puede disfrutar de alguna noche libre en pareja.

Las parejas del estudio, particularmente, las más jóvenes, muestran un alto nivel de compromiso y colaboración. La mayoría de ellas han negociado quién lleva a los hijos al colegio y quién los recoge en cada momento, comparten la obligación de ir de compras y la responsabilidad de hacer pequeñas tareas domésticas. Pero ninguna de estas parejas ha invertido completamente los roles tradicionales, es decir, las mujeres siguen realizando las tareas de planificación y control esenciales en el hogar ${ }^{30}$. Ni siquiera sucede en aquellas parejas en las cuales las expectativas de las mujeres profesionales han impulsado decisiones esenciales para sus familias como el cambio de residencia a otra ciudad o a otro país.

Las relaciones de género tradicionales siguen vigentes. Esto es lo que se deduce del análisis de sus estrategias familiares. Por ejemplo, no hay discusión sino negociación cuando el cabeza de familia es un hombre y decide que toda la familia ha de marchar. En cambio, analizando las experiencias de las mujeres investigadoras ${ }^{31}$, cuando una mujer lidera la decisión de marchar, la negociación es más larga e implica a más agentes (los hijos, la familia extensa, etc.). El convencimiento de que es imposible subvertir completamente los roles de género puede estar reforzando la idea de algunas mujeres de que es mejor simplemente renunciar a cualquier responsabilidad relacionada con la maternidad y la familia.

30. Hochschild, Arlie y Machung, Anne, op. cit.

31. GonZÁlez Ramos, Ana M. y Vergés Bosch, Núria, op. cit.

Feminismo/s 23, junio 2014, pp. 183-205 
Por otra parte, algunas investigadoras consideran que una de las ventajas de trabajar en la academia es la flexibilidad que proporciona el trabajo científico. Esta afirmación, expresada particularmente por las investigadoras de ciencias sociales y humanidades, es cuanto menos paradójica, ya que la ciencia impone un ritmo de trabajo muy intenso, que reduce el tiempo libre y funde los tiempos de trabajo y descanso. Lo que estas mujeres valoran positivamente es la posibilidad de gestionar el tiempo y la autonomía frente a una organización institucional inflexible de la jornada laboral. La auto-gestión del tiempo ha sido un recurso fundamental para ellas, especialmente durante la etapa de cuidado de los hijos menores de edad, y es en la actualidad un modelo de gestión su cotidianidad. A medida que las necesidades de una esfera u otra de sus vidas cambian (según la carga de trabajo, la edad de los hijos, etc.) han gestionado de manera diferente su tiempo de trabajo y de descanso y ocio.

En sus discursos, las estrategias individuales superan cualquier influencia positiva que las políticas públicas puedan haber desempeñando en sus trayectorias vitales. A la pregunta sobre cómo han logrado mantener el ritmo del trabajo científico y la familia, las investigadoras responden que lo han logrado mediante la auto-organización, con la ayuda de sus parejas (cuando son colaboradoras en el hogar) y educando a sus hijos con actitudes de responsabilidad y autonomía personal. En menor medida aluden a las medidas de conciliación, permisos de paternidad u otras políticas. Las más veteranas mencionan que esas medidas ni siquiera existían en el pasado. Más que las conquistas laborales, sociales y de igualdad, ellas insisten en su capacidad de gestión de la cotidianidad.

Por el contrario, las medidas de igualdad que en principio deberían favorecer las trayectorias laborales de las investigadoras son mencionadas, precisamente, cuando se ha producido una situación de discriminación clara (no pudo acogerse a un permiso de paternidad en su momento por su situación de precariedad laboral); o, cuando se ha conseguido cambiar una situación desfavorable para las mujeres, por ejemplo, cuando ha sido la primera vez que una mujer disfrutó de las horas de maternidad en un departamento altamente masculinizado. Pese a las mejoras evidentes que proporcionan las políticas sociales y de igualdad, ellas remarcan su agencia en la vida privada. De hecho, cada una de estas mujeres ha tenido que elaborar una estrategia planificada y cambiante que les permitiera conjugar sus aspiraciones profesionales con sus obligaciones familiares en un contexto laboral muy competitivo. 


\section{Principales resultados}

A lo largo de este trabajo hemos comprobado que el cambio de valores sociales y, sobre todo, las condiciones de trabajo que impone la ciencia provoca cambios sustanciales en las estructuras familiares de las mujeres investigadoras. Su fuerte orientación profesional no les ha impedido renunciar a sus familias. Quienes mantienen esta postura, han desarrollado previamente sus carreras y una vez lograda cierta estabilidad, la familia ha ocupado un primer plano. Para conciliar ambas esferas, las mujeres desarrollan estrategias familiares y de género diferentes entre sí. En este trabajo hemos comprobado que los factores más importantes son sus estilos de vida o valores sobre la familia y la profesión, su situación económica y entorno económico, y su estructura familiar, es decir, el perfil de su pareja, la existencia de hijos o no, y las posibilidades que tienen de gestionar el tiempo.

Debido a características culturales de la sociedad española, las mujeres científicas presentan un fuerte compromiso con la familia pero, al mismo tiempo, una valoración de la actividad profesional bastante independiente de sus condicionantes personales no completamente separada de los roles sociales tradicionales. Los casos de abandono de la profesión no se producen por cuestiones personales o familiares, ni siquiera por tener experiencias de discriminación o fracaso, sino porque se valora negativamente la organización científica y su modelo de éxito. En cambio, sí se produce una ralentización de las carreras, interrupciones o menores logros profesionales.

La elección de las parejas es clave para el desarrollo profesional de las científicas, aunque no es el único factor determinante. Hemos descrito varios tipos de relaciones donde las mujeres han establecido un compromiso profesional y personal con sus parejas. Otro tipo de parejas colaboradoras supone una relación de mentorazgo, donde la pareja más joven se beneficia de la experiencia de la de más edad o de su mentor. En otras relaciones, la existencia de una pareja científica supone un letargo para las mujeres, porque renuncian a seguir el ritmo competitivo de la academia o sus áreas de conocimiento. A veces, simplemente, son eclipsados por los logros de sus parejas. Finalmente, las parejas adversarias que impedirían las trayectorias femeninas son difícilmente detectables entre las investigadoras más jóvenes. Ellas hablan de una ex pareja con la que ya no compartían planes comunes y que abandonaron. Por tanto, el divorcio puede ser una manera de escapar de una pareja obstaculizadora de las trayectorias profesionales de las mujeres científicas.

Respecto a su estrategia familiar de conciliación, el nivel socio-económico que se disfruta es decisivo para organizar la cotidianidad de estas familias. En segundo lugar, estarían la red de recursos públicos y la organización de la 
familia en un equipo de colaboradores donde la principal responsable sigue siendo la mujer. Por ello, la auto-organización es un principio especialmente valorado por las investigadoras. La gestión de los tiempos es cambiante a lo largo del tiempo, dependiendo de las características de las familias y de los compromisos profesionales. Es el tiempo de ocio, descanso e intimidad con la pareja el más afectado por esta situación desfavorable puesto que estas mujeres muestran un alto grado de compromiso, tanto respecto a su trabajo como a sus familias.

La actitud de los hombres que forman parte de estas parejas ha cambiado, pero no tan sustancial ni profundamente como sería deseable. No sólo hay un mayor grado de colaboración sino que la toma de decisión es más igualitaria. Sin embargo, muchos rasgos tradicionales siguen estando presentes y representan una desventaja para las mujeres como, por ejemplo, la justificación de la toma de decisiones y el que la responsabilidad última de la organización familiar siga recayendo sobre ellas. Así pues, esos cambios que podríamos suponer ventajosos para las mujeres altamente cualificadas que tienen más recursos para establecer relaciones de igualdad con sus parejas, sin embargo, no se han incorporado sino en pequeñas dosis a las familias de las investigadoras incluidas en nuestro estudio. Por tanto, podemos seguir contando con las barreras familiares y sociales, como obstáculos al progreso y reconocimiento del talento femenino.

Sin embargo, este estudio debería realizarse posterioridad o considerando los cambios que se están produciendo en las familias científicas más jóvenes. Una limitación de este estudio es que las mujeres de mayor edad están «arrastrando» las conclusiones de nuestro estudio hacia posiciones más familistas y tradicionales. También sería deseable que este estudio incluyera opiniones de los hombres (ya sean científicos o no) respecto a la carrera de sus parejas y la gestión del tiempo común en el hogar. Ello puede constituir una futura línea de investigación muy fructífera desde el punto de vista de las masculinidades.

\section{Agradecimientos}

Este trabajo ha sido elaborado gracias a la financiación del Ministerio de Ciencia e Innovación CSO2009-09003. Parte de esta información ha sido presentado en el IX Congreso Iberoamericano de Ciencia, Tecnología y Género, celebrado en Sevilla en 2012. Nuestro agradecimiento a todas las compañeras que han participado en este estudio, así como a las que han leído previamente versiones preliminares de este trabajo. 


\section{Referencias bibliográficas}

BAgILHOLE, Barbara y GOODE, Jackie «The Contradiction of the Myth of Individual Merit, and the Reality of a Patriarchal Support System in Academic Careers: A Feminist Investigation, European Journal of Women's Studies», 8, 2, 161-180, 2001.

Barral, M. J., Delgado, I., Turrado, T. y Magallón, C.: «Life Paths of Successful Women Scientists in Spain», Géneros, Multidisciplinary Journal of Gender Studies, 3 (1): 351-372.

Boyle, Paul J. et al (ed.) «Moving and union dissolution», Demography, 45, 1, 209-222, February 2008.

CABRÉ, Monstserrat: «La Ciencia de las mujeres en la Edad Media. Reflexiones sobre la autoría femenina», en C. SEGURA GRAIÑo (ed.): La voz del silencio II, Madrid, Almudayna, 1993.

Etzkowitz Henry, Kemelgor, Carol y Uzzi, Brian: Athena Unbound: The Advancement of Women in Science and Technology, Cambridge University Press, Cambridge, 2000.

ETzKowitz, Henry y RANGA, Marina: «Gender dynamics in science and technology: From the "Leaky Pipeline" to the "Vanish Box"», Brussels Economic Review, Special Issue «Beyond the leaky pipeline - Challenges for research on gender and science», 54 (2/3): 131-148, Summer-Autumn 2011.

EVERGETI, Venettia y RYAN, Louise: «Negotiating transnational caring practices among migrant families». En KRALER, Albert et al. (eds.) Gender, Generations and the Family in International Migration. Utrech: Amsterdam University Press, 2011.

Fox, Mary F: «Gender, Family Characteristics, and Publication Productivity among Scientists», Social Studies of Science, 35 (1), 131-150, 2005.

GARCÍA DE LEÓN, M. Antonia et al.: Las académicas: profesorado universitario y género, Madrid: Instituto de la Mujer, 2001.

GARCÍA DE CORTÁZAR, M. Luisa et al.: Mujeres y hombres en la ciencia española. Una investigación empírica, Madrid: Instituto de la Mujer, 2006.

GONZÁlez RAmos, Ana M.: «Victoria y la ciencia: El papel de las mujeres en la ciencia contemporánea» en CASTAÑO, Cecilia (dir.): Mujeres Construyendo la Sociedad del Conocimiento: Ciencia, Tecnología y Género, Barcelona: Editorial Aresta, 2014.

GONZÁlez RAmos, Ana M. y Vergés BOSCH, Núria: «International mobility of women in S\&T careers: Shaping plans for personal and professional purposes», Gender, Place and Culture, 20, 5, 613-629, 2013.

GREEN, Andy E.: «A question of compromise? Case study evidence on the location and mobility strategies of dual career households», Regional Studies, 31, 7, 641-57, 1997. 
HERTZ, Rosanna: More Equal Than Others: Women and Men in Dual-Career Marriages, University of California Press, Berkeley, CA, 1986.

Hochschild, Arlie y Machung, Anne: The Second Shift, New York: Penguin, 1989.

KulU, Hill: «Migration and Fertility: Competing Hypotheses Re-examined», European Journal of Population, 21, 51-87, 2005.

LYON, Dawn y WOODWARD, Alison E. «Gender and Time at the Top: Cultural Construction of Time in High-Level Careers and Homes», European Journal of Women's Studies, 11, 2, 205-221, 2004.

Magallón, Carmen: Pioneras españolas en las ciencias. Las mujeres del Instituto Nacional de Física y Química, Madrid, CSIC, 1998.

MiQUeO, C. et al.: «Del análisis crítico a la autoridad femenina en la ciencia, Feminismo/s, 1, 195-216, junio 2003.

Monnosson, Emily ed.: Motherhood, the elephant in the laboratory, Cornell University Press, 2008.

NAS Committee on Science, Engineering and Public Policy: Beyond bias and barriers: Fulfilling the potential of women in academic science and engineering, Washington, D.C., National Academies Press, 2007.

O'NeIL, Deborah A. y Bilimoria, Diana «Women's career development phases. Idealism, endurance, and reinvention, Career Development International, 10, 3, 168-189, 2005.

Ortíz Gómez, Teresa y BeCerra Conde, Gloria (eds.): Mujeres de ciencia. Mujer, feminismo y ciencias naturales, experimentales y tecnológicas, Granada, Universidad de Granada, 1996.

Pérez Sedeño, Eulalia (ed.): Las mujeres en el Sistema de Ciencia y Tecnología, Estudios de casos, OEI, 2001.

Pycior, Helena, M., G. SLACK, Nancy y ABIR-AM, Pnina G. ed.: Creative Couples in the Sciences, Rutgers, 1996.

Rossiter, Margaret W.: «The Matthew Matilde Effect in Science», Social Studies of Science, vol. 23 (2): 325-341, 1993.

SANTESMASES, M. Jesús: Mujeres científicas en España (1940-1970). Profesionalización y modernización social, Madrid, Instituto de la Mujer, Ministerio de Trabajo y Asuntos Sociales, 2000.

Schiebinger, Londa, DAvis Henderson, Andrea y Gilmartin, Shannon K.: DualCareer Academic Couples. What Universities Need to Know, Michelle R. Clayman Institute for Gender Research at Stanford University, Stanford, CA, 2008.

UNIDAD DE MUJERES Y CIENCIA: Libro Blanco. Situación de las Mujeres en la Ciencia Española, Madrid, Ministerio de Ciencia e Innovación, 2011.

Vergés Bosch, Núria y GonzÁlez Ramos, Ana M.: «Beyond the Work-Life Balance: Family and International Mobility of the Highly Skilled», Sociología 
y Tecnociencia. Revista Digital de Sociología del Sistema Tecnocientífico, 3, 55-76, 2013.

WAGNER, Izabela «Career Coupling: Career Making in the Elite World of Musicians and Scientists, Qualitative Sociology Review, II, 3, 78-98, December 2006.

XIE, Yie y Shauman, Kimberlee A.: Women in Science: Career Processes and Outcomes, Harvard University Press, Cambridge, Massachusetts, 2003. 